\title{
A METRIC SPACE ASSOCIATED WITH A PROBABILITY SPACE
}

\section{KEITH F. TAYLOR and XIKUI WANG}

\author{
Department of Mathematics \\ University of Saskatchewan \\ Saskatoon, Sask. Canada S7N 0W0 \\ (Received January 7, 1992)
}

\begin{abstract}
For a complete probability space $(\Omega, \Sigma, P)$, the set of all complete sub- $\sigma$-algebras of $\Sigma, S(\Sigma)$, is given a natural metric and studied. The questions of when $S(\Sigma)$ is compact or connected are awswered and the important subset consisting of all continuous sub- $\sigma$-algebras is shown to be closed. Connections with Christensen's metric on the von Neumann subalgebras of a Type $\mathrm{II}_{1}$-factor are briefly discussed.
\end{abstract}

KEY WORDS AND PHRASES. $\sigma$-algebras, conditional expectations, metric space, von Neumann algebra.

1992 AMS SUBJECT CLASSIFICATION CODES. Primary: 28A05, 28A20. Secondary: $46 \mathrm{~L} 10$.

\section{INTRODUCTION.}

Let $(\Omega, \Sigma, P)$ be a complete probability space and let $S(\Sigma)$ denote the set of all complete sub- $\sigma$ algebras of $\Sigma$. We will define a metric $d$ on $S(\Sigma)$ and investigate some topological properties of the resulting metric space $(S(\Sigma), d)$. In [1], Boylan introduced a metric $d^{\prime}$ on $S(\Sigma)$ for the purpose of studying convergence properties of the conditional expectation operators defined by varying sub- $\sigma$ algebras of $\Sigma$. Our metric turns out to be equivalent to Boylan's and seems to be more convenient for study using functional analytic methods.

We prove, in section 3 , that $(S(\Sigma), d)$ is compact if and only if $\Sigma$ is purely atomic. We also show that $(S(\Sigma), d)$ is connected if and only if $\Sigma$ has at most one atom.

In section 4 , we consider the continuous sub- $\sigma$-algebras of $\Sigma$ and show that they form a closed nowhere dense subset of $(S(\Sigma), d)$.

There is a close analogy between probability spaces and von Neumann algebras with a faithful finite normal trace. In fact, our definition of $d$ is modeled on a metric defined by Christensen [2] on the set of all von Neumann subalgebras of a Type II $_{1}$-factor. Christensen's metric has been useful in the study of the index in $\mathrm{II}_{1}$-factors (see [3] and [5]). We give a short discussion of a common generalization of our metric and Christensen's in section 5 .

\section{THE METRICS.}

In this section, we define the metric $d$ and Boylan's metric $d^{\prime}$ and show that they are equivalent. If $\Sigma_{0} \in S(\Sigma)$, then let $L^{\infty}\left(\Sigma_{0}\right)=L^{\infty}\left(\Omega, \Sigma_{0}, P\right)$ be considered as a closed subspace of $L^{\infty}(\Omega, \Sigma, P)$ in the natural way. Since bounded functions are square intergrable on a probability space, we may consider $L^{\infty}\left(\Sigma_{0}\right)$ as a (non-closed) subspace of the Hilbert space $L^{2}(\Omega, \Sigma, P)$. It is easy to check that the unit ball in $L^{\infty}\left(\Sigma_{0}\right)$ is closed in $L^{2}(\Omega, \Sigma, P)$. The metric $d$ is essentially the Hausdorff metric on the unit balls in the $L^{2}$-norm. Let 


$$
L^{\infty}\left(\Sigma_{0}\right)_{1}=\left\{f \in L^{\infty}\left(\Sigma_{0}\right):\|f\|_{\infty} \leq 1\right\}
$$

For $\Sigma_{1}, \Sigma_{2} \in S(\Sigma)$, let

$$
d\left(\Sigma_{1}, \Sigma_{2}\right)=\max \left\{\sup _{f \in L^{\infty}\left(\Sigma_{1}\right)_{1}} \inf _{g \in L^{*}\left(\Sigma_{2}\right)_{1}}\|f-g\|_{2}, \sup _{g \in L^{\infty}\left(\Sigma_{2}\right)_{1}} \inf _{f \in L^{\infty}\left(\Sigma_{1}\right)_{1}}\|f-g\|_{2}\right\} .
$$

It is easy to check that $d$ is a metric on $S(\Sigma)$.

For the metric $d^{\prime}$, we use a definition due to Rogge [6] which is a slight variation on Boylan [1]. For $\Sigma_{1}, s_{2} \in S(\Sigma)$, let

$$
d^{\prime}\left(\Sigma_{1}, \Sigma_{2}\right)=\sup _{A \in \Sigma_{1}} \inf _{B \in \Sigma_{2}} P(A \Delta B) \vee \sup _{B \in \Sigma_{2}} \inf _{A \in \Sigma_{1}} P(A \Delta B)
$$

(Boylan's metric $d^{*}$ has $\vee$ replaced by + and clearly $\frac{1}{2} d^{*} \leq d^{\prime} \leq d^{*}$, so $d^{\prime}$ and $d^{*}$ are essentially the same). The arguments in $[\mathbf{1}]$ show that $\left(S(\Sigma), d^{\prime}\right)$ is a complete metric space.

For any $\Sigma_{0} \in S(\Sigma)$, let $\epsilon^{\Sigma_{0}}$ denote the orthogonal projection of $L^{2}(\Omega, \Sigma, P)$ onto $L^{2}\left(\Omega, \Sigma_{0}, P\right)$, considered as a subspace of $L^{2}(\Sigma)$. Let $E^{\Sigma_{0}}$ denote the restriction of $e^{\Sigma_{0}}$ to $L^{\infty}(\Sigma)$. As is well known, $e^{\Sigma_{0}}$ and $E^{\Sigma_{0}}$ are restrictions of the conditional expectation mapping of $L^{1}(\Omega, \Sigma, P)$ onto $L^{1}\left(\Omega, \Sigma_{0}, P\right)$. We will use any of the well known special properties of conditional expectation without giving references.

We now consider the relationship between the metric $d$ and $d^{\prime}$.

THEOREM 1. For any $\Sigma_{1}, \Sigma_{2} \in S(\Sigma)$,

$$
d^{\prime}\left(\Sigma_{1}, \Sigma_{2}\right) \leq d\left(\Sigma_{1}, \Sigma_{2}\right) \leq 2 \sqrt{2 d^{\prime}\left(\Sigma_{1}, \Sigma_{2}\right)\left(1-d^{\prime}\left(\Sigma_{1}, \Sigma_{2}\right)\right)}
$$

Thus $d^{\prime}$ and $d$ are equivalent metrics.

PROOF. To prove the left hand inequality, fix $A \in \Sigma_{1}$. It is shown in 2.1 of [4] that $\inf _{B \in \Sigma_{2}} P(A \Delta B)$ is achieved at the set $C=\left\{E^{\Sigma_{2}}\left(\chi_{A}\right)>\frac{1}{2}\right\}$, where $\chi_{A}$ is the indicator function of the set $A$. Some elementary manipulations show that

Now $\left\|\frac{1}{2}-\chi_{A}\right\|_{1}=\frac{1}{2}$, so

$$
P(A \Delta C)=\frac{1}{2}-\int_{\Omega}\left|\frac{1}{2}-E^{\Sigma_{2}}\left(\chi_{A}\right)\right| d P
$$

$$
P(A \Delta C)=\left\|\frac{1}{2}-\chi_{A}\right\|_{1}-\left\|\frac{1}{2}-E^{\Sigma_{2}}\left(\chi_{A}\right)\right\|_{1} \leq\left\|\chi_{A}-E^{\Sigma_{2}}\left(\chi_{A}\right)\right\|_{1} \leq\left\|\chi_{A}-E^{\Sigma_{2}}\left(\chi_{A}\right)\right\|_{2}
$$

Thus $\inf _{B \in \Sigma_{2}} P(A \Delta B) \leq d\left(\Sigma_{1}, \Sigma_{2}\right)$, for all $A \in \Sigma_{1}$. Symmetric arguments apply for $\Sigma_{2}$, which gives $d^{\prime}\left(\Sigma_{1}, \Sigma_{2}\right) \leq d\left(\Sigma_{1}, \Sigma_{2}\right)$.

For the right inequality, note that, for any $f \in L^{\infty}\left(\Sigma_{1}\right)_{1}, E^{\Sigma_{1}}(f)=f$ and $E^{\Sigma_{2}}(f)$ is the $\left(L^{2}\right.$. norm) closest element of $L^{\infty}\left(\Sigma_{2}\right)_{1}$ to $f$. Thus,

$$
\sup _{f \in L^{\infty}\left(\Sigma_{1}\right)_{1}} \inf _{g \in L^{\infty}\left(\Sigma_{2}\right)_{1}}\|f-g\|_{2}=\sup _{f \in L^{\infty}\left(\Sigma_{1}\right)_{1}}\left\|f-E^{\Sigma_{2}}(f)\right\|_{2} \leq \sup _{f \in L^{\infty}(\Sigma)_{1}}\left\|E^{\Sigma_{1}}(f)-E^{\Sigma_{2}}(f)\right\|_{2}
$$

Similarly for the other term in $d\left(\Sigma_{1}, \Sigma_{2}\right)$, so we have the inequality

$$
d\left(\Sigma_{1}, \Sigma_{2}\right) \leq \sup _{f \in L^{\infty}(\Sigma)_{1}}\left\|E^{\Sigma_{1}}(f)-E^{\Sigma_{2}}(f)\right\|_{2}
$$

However, for any $f \in L^{\infty}(\Sigma)_{1}$, the function $f^{\prime}=\frac{1}{2}(1+f)$ takes values in $[0,1]$ almost everywhere 
and theorem 3 in [6] shows that

$$
\left\|E^{\Sigma_{1}}\left(f^{\prime}\right)-E^{\Sigma_{2}}\left(f^{\prime}\right)\right\|_{2} \leq \sqrt{2 d^{\prime}\left(\Sigma_{1}, \Sigma_{2}\right)\left(1-d^{\prime}\left(\Sigma_{1}, \Sigma_{2}\right)\right)}
$$

Thus,

$$
\left\|E^{\Sigma_{1}}(f)-E^{\Sigma_{2}}(f)\right\|_{2} \leq 2 \sqrt{2 d^{\prime}\left(\Sigma_{1}, \Sigma_{2}\right)\left(1-d^{\prime}\left(\Sigma_{1}, \Sigma_{2}\right)\right)}
$$

This completes the proof of the theorem.

COROLLARY. The identity map is a homeomorphism of $\left(S(\Sigma), d^{\prime}\right)$ with $(S(\Sigma), d)$.

REMARK 1. Not only are $d$ and $d$ equivalent in the sense that they define the same open scts in $S(\Sigma)$, but the same sequences are Cauchy in each of $(S(\Sigma), d)$ and $\left(S(\Sigma), d^{\prime}\right)$. Since $\left(S(\Sigma), d^{\prime}\right)$ is complete, we have that $(S(\Sigma), d)$ is a complete metric space.

REMARK 2. The right hand inequality in theorem 1 cannot be improved to one of the form $d \leq k d^{\prime}$ for some positive constant $k$, as is shown by the following example.

EXAMPLE. Let $\Omega=[0,1]$, with $P$ denoting Lebesgue measure on $\Sigma$, the Lebesgue measurable subsets of $[0,1]$. For each $a>0$, let $\Sigma_{u}$ denote the $\sigma$-algebra generated by an atum $[0, a]$ and $\Sigma \cap(a, 1]=\{B \in \Sigma: B \subseteq(a, 1]\}$. For any $A \in \Sigma$, let $B_{1}=A \cap(a, 1]$ and $B_{2}=[0, a] \cup(A \cap(a, 1])$. Then $B_{1}, B_{2} \in \Sigma_{a}$ and either $P\left(A \Delta B_{1}\right)$ or $P\left(A \Delta B_{2}\right)$ is less than or equal to $\frac{a}{2}$. If $A=\left[0, \frac{a}{2}\right]$, then $P(A \Delta B) \geq \frac{a}{2}$, for all $B \in \Sigma_{a}$. Thus $d^{\prime}\left(\Sigma_{a}, \Sigma\right)=\frac{a}{2}$.

To compute $d\left(\Sigma_{a}, \Sigma\right)$, note that, for any $f \in L^{\infty}(\Sigma)_{1}$, the function $f_{X(a, 1]}$ is in $L^{\infty}\left(\Sigma_{a}\right)_{1}$ and $\left\|f-f \chi_{(a, 1]}\right\|_{2} \leq \sqrt{a}$. On the other hand, if $f=\chi_{\left[0, \frac{a}{2}\right]}-\chi_{\left(\frac{a}{2}, a\right]}$, then $E^{\Sigma_{a}}(f)=0$ almost everywhere. This implies that $\|f-g\|_{2} \geq\|f\|_{2}=\sqrt{a}$, for any $g \in L^{\infty}\left(\Sigma_{a}\right)_{1}$. Thus $d\left(\Sigma_{a}, \Sigma\right)=\sqrt{a}$.

With $\Sigma_{1}=\Sigma_{a}$ and $\Sigma_{2}=\Sigma$, the inequalities in theorem 1 become $\frac{a}{2} \leq \sqrt{a} \leq 2 \sqrt{\frac{a}{2}-\left(\frac{a}{2}\right)^{2}}(\leq$ $\sqrt{2 a})$. Thus no $k$ exists with $d\left(\Sigma_{a}, \Sigma\right) \leq k d^{\prime}\left(\Sigma_{a}, \Sigma\right)$ for all $a \in(0,1)$.

\section{THE MAIN RESULTS.}

We consider the metric space $(S(\Sigma), d)$ and establish the following two theorems. Since $\left(S(\Sigma), d^{\prime}\right)$ is homeomorphic to $(S(\Sigma), d)$, these characterizations hold for either metric.

THEOREM 2. $(S(\Sigma), d)$ is compact if and only if $\Sigma$ is purely atomic.

THEOREM 3. $(S(\Sigma), d)$ is connected if and only if $\Sigma$ has at most one atom.

Before proving these theorems, we need the following lemma which is a folklore result of measure theory and can be proven with an easy application of Zorn's lemma.

LEMMA If $(X, \mathcal{F}, \mu)$ is a finite measure space with no atoms, then there exists a map $F$ : $[0,1] \rightarrow \mathcal{F}$ such that $F(s) \subseteq F(t)$ and $\mu(F(t))=t \mu(X)$ for all $s, t \in[0,1]$ with $s \leq t$.

We will use the notation, for $A \in \Sigma$ and $\Sigma_{0} \in S(\Sigma)$,

$$
A \bigcap \Sigma_{0}=\left\{A \bigcap B: B \in \Sigma_{0}\right\}
$$

PROOF OF THEOREM 2. Assume that $(\Omega, \Sigma, P)$ is not completely atomic. Let $\Omega_{c}$ and $\Omega_{a}$ be the continuous and atomic parts of $\Omega$ respectively. We are assuming $P\left(\Omega_{c}\right)>0$. Let $F:[0,1] \rightarrow \Sigma$ be such that $F(t) \subseteq \Omega_{c}, P(F(t))=t P\left(\Omega_{c}\right)$, and $F(s) \subseteq F(t)$ for $s, t \in[0,1], s \leq t$. Define $F[s, t]=F(t) \backslash F(s)$ and let 


$$
A_{n}=\bigcup\left\{F\left[(k-1) / 2^{n-1},(2 k-1) / 2^{n}\right]: k=1,2, \ldots, 2^{n-1}\right\}
$$

for each $n=1,2, \ldots$ Then $P\left(A_{n}\right)=P\left(\Omega_{c}\right) / 2$, for each $n$ and $P\left(A_{n} \Delta A_{m}\right)=P\left(\Omega_{c}\right) / 2$, for $n \neq m$. Let $\Sigma_{n}$ debote the $\sigma$-algebra generated by $A_{n} \cap \Sigma$ and the atom $\Omega \backslash A_{n}$, for each $n$. Let $f$ denote the indicator function of $\Omega \backslash A_{n}$. Then $f \in L^{\infty}\left(\Sigma_{n}\right)_{1}$ and for any $g \in L^{\infty}\left(\Sigma_{m}\right)_{1}$, if $m \neq n$, then $g$ differs from $f$ by at least $\frac{1}{2}$ on either $\left(\Omega \backslash A_{m}\right) \cap A_{n}$ or $\left(\Omega \backslash A_{m}\right) \cap\left(\Omega \backslash A_{n}\right)$. Each of these sets has probability $P\left(\Omega_{c}\right) / 4$. Thus $\|f-g\|_{2}^{2} \geq P\left(\Omega_{c}\right) / 16$, which implies $d\left(\Sigma_{n}, \Sigma_{m}\right) \geq \sqrt{P\left(\Omega_{c}\right)} / 4$, if $n \neq m$. Hence, the sequence $\left(\Sigma_{n}\right)_{n=1}^{\infty}$ has no cluster points and $S(\Sigma)$ is not compact.

Conversely, suppose $(\Omega, \Sigma, P)$ is completely atomic. Since $(S(\Sigma), d)$ is complete, compactness will follow if we show that $S(\Sigma)$ is totally bounded. If $\Sigma$ has only a finite number of atoms, then $S(\Sigma)$ is a finite set and obviously compact. So suppose $\left\{A_{1}, A_{2}, \ldots\right\}$ are disjoint atoms generating $\Sigma$. For $\epsilon>0$, choose $N$ such that $\sum_{n=N+1}^{\infty} P\left(A_{n}\right)<\epsilon^{2}$. There are only a finite number of algebras of sets contained in the algebra generated by $\left\{A_{1}, \ldots, A_{N}\right\}$. Let $\mathcal{A}_{1}, \ldots, \mathcal{A}_{k}$ denote these algebras. For $1 \leq j \leq k$, let $\Sigma$, denote the $\sigma$-algebra generated by $\mathcal{A}_{j}$ and $\left\{A_{N+1}, A_{N+2}, \ldots\right\}$. Let $A=\bigcup_{n=1}^{N} A_{n}$. For any $\Sigma^{\prime} \in S(\Sigma), A \cap \Sigma^{\prime}=\mathcal{A}_{j}$, for some $j, 1 \leq j \leq k$. Then $\Sigma^{\prime} \subseteq \Sigma$, and it is easy to check that $d\left(\Sigma^{\prime}, \Sigma_{j}\right)<\epsilon$. Therefore, $S(\Sigma)$ is covered by the finite set of $\epsilon$-balls centered at $\Sigma_{j}, 1 \leq j \leq k$. Since $\epsilon>0$ was arbitrary, $S(\Sigma)$ is totally bounded.

PROOF OF THEOREM 3. Suppose $A$ and $B$ are two distinct atoms in $\Sigma$. Let $S_{1}$ denote the set of all $\Sigma_{0}$ in $S(\Sigma)$ which have an atom containing both $A$ and $B$, let $S_{2}$ denote the set of all $\Sigma_{0}$ in $S(\Sigma)$ which have two disjoint atoms containing $A$ and $B$, respectively. It is easy to see that $S(\Sigma)=S_{1} \cup S_{2}$. Let $\Sigma_{1} \in S_{1}$ and $\Sigma_{2} \in S_{2}$. Let $A^{\prime}$ and $B^{\prime}$ be disjoint atoms of $\Sigma_{2}$ containing $A$ and $B$, respectively. Let $C$ be an atom of $\Sigma_{1}$ which contains $A \cup B$. Let $f=\chi_{A^{\prime}}-\chi_{B^{\prime}} \in L^{\infty}\left(\Sigma_{2}\right)_{1}$. For any $g \in L^{\infty}\left(\Sigma_{1}\right)_{1}, g=a$, almost everywhere on $C$, for some constant $a,|a| \leq 1$. Thus

$$
\begin{aligned}
\|f-g\|_{2} & \geq \sqrt{\int_{A}|f-g|^{2} d P+\int_{B}|f-g|^{2} d P} \\
& =\sqrt{(1-a)^{2} P(A)+(1+a)^{2} P(B)} \\
& \geq \sqrt{2 \min \{P(A), P(B)\}}
\end{aligned}
$$

Therefore, $d\left(\Sigma_{1}, \Sigma_{2}\right) \geq \sqrt{2 \min \{P(A), P(B)\}}$, for all $\Sigma_{1} \in S_{1}$ and $\Sigma_{2} \in S_{2}$. Notice also that the trivial $\sigma$-algebra $\Sigma_{\tau}=\{\Omega, \emptyset\} \in S_{1}$ and $\Sigma \in S_{2}$. So $S_{1}$ and $S_{2}$ are nonempty disjoint open subsets of $S(\Sigma)$ with union $S(\Sigma)$. Hence, $S(\Sigma)$ is not connected if there are two or more atoms in $\Sigma$.

Conversely, suppose $(\Omega, \Sigma, P)$ has at most one atom. If there is an atom, call it $A$ and if there is no atom, let $A=\emptyset$. If $P(A)=1$, then $S(\Sigma)$ is a one point space, which is connected. So assume $P(\Omega \backslash A)>0$. We will show that any $\Sigma_{0} \in S(\Sigma)$ can be connected to $\Sigma$ by an arc in $S(\Sigma)$.

First assume $\Sigma_{0} \in S(\Sigma)$ and $\Sigma_{0}$ is completely atomic, generated by the disjoint atoms $A_{0}, A_{1}, \ldots$ (a finite or infinite collection). We may assume $A \subseteq A_{0}$. For each $n \geq 1$, by the lemma, there exists $F_{n}:[0,1] \rightarrow A_{n} \cap \Sigma$ such that $P\left(F_{n}(t)\right)=t P\left(A_{n}\right)$ and $F_{n}(s) \subseteq F_{n}(t)$ if $s, t \in[0,1]$ with $s \leq t$. Similarly, let $F_{0}:[0,1] \rightarrow A_{0} \cap \Sigma$ be such that $F_{0}$ is increasing and $P\left(F_{0}(t)\right)=P(A)+$ $t P\left(A_{0} \backslash A\right)$, for all $t \in[0,1]$. For each $t \in[0,1]$, define $\Sigma(t) \in S(\Sigma)$ as the $\sigma$-algebra generated by $\left\{F_{n}(t) \cap \Sigma, A_{n} \backslash F_{n}(t): n=0,1,2, \ldots\right\}$. It is clear that $\Sigma(0)=\Sigma_{0}, \Sigma(1)=\Sigma$ and $\Sigma(s) \subseteq \Sigma(t)$ if $s \leq t$. Fix $s, t \in[0,1]$ with $s \leq t$. 
For any $f \in L^{\infty}(\Sigma(t))_{1}$ and for each $n=0,1,2, \ldots, f$ is constant, say $a_{n}$, almost everywhere on the atom $A_{n} \backslash F_{n}(t)$ in $\Sigma(t)$. Define $g \in L^{\infty}(\Sigma(s))_{1}$, by making $g=a_{n}$ on $A_{n} \backslash F_{n}(s)$ and $g=f$ on $F_{n}(s)$, for $n=0,1,2, \ldots$ Then $f$ and $g$ differ only on $\bigcup\left(F_{n}(t) \backslash F_{n}(s)\right)$ and there by at most 2 . Now

$$
P\left(\bigcup\left(F_{n}(t) \backslash F_{n}(s)\right)\right)=(t-s)\left[P\left(A_{0} \backslash A\right)+P\left(A_{1}\right)+P\left(A_{2}\right)+\ldots\right]
$$

Thus $\|f-g\|_{2} \leq 2 \sqrt{t-s}$.

This implies that $d(\Sigma(s), \Sigma(t)) \leq 2 \sqrt{t-s}$. Therefore, any atomic element $\Sigma_{0}$ of $S(\Sigma)$ is connected by an arc to $\Sigma$.

If $\Sigma_{0}$ is not completely atomic, let $\Omega=\Omega_{c} \cup \Omega_{a}$, where $\Omega_{c} \cap \Sigma_{0}$ is continuous and $\Omega_{a} \cap \Sigma_{0}$ is atomic. Let $\Sigma_{1}$ be the atomic $\sigma$-algebra generated by an atom equal to $\Omega_{c}$ and $\Omega_{a} \cap \Sigma_{0}$. By a method similar to the above, but just working with $\Omega_{c}$, an arc from $\Sigma_{1}$ to $\Sigma_{0}$ can be found. Also, $\Sigma_{1}$ being atomic is connected by an arc to $\Sigma$. Thus any element $\Sigma_{0}$ of $S(\Sigma)$ can be connected by an arc to $\Sigma$. Therefore $S(\Sigma)$ is connected, in fact, arcwise connected if $(\Omega, \Sigma, P)$ has at most one atom.

The two theorems of this section show that there is some meaningful connection between the topology of $S(\Sigma)$ and the structure of the original probability space. Since this topology is really an embodiment of the equiconvergence property for conditional expectations, we feel that $S(\Sigma)$ with this metric $d$ (or if one prefers $d^{\prime}$ ) will provide a good locale for studying conditional expectations.

\section{CONTINUOUS SUB- $\sigma$-ALGEBRAS.}

Let $S_{c}(\Sigma)=\left\{\Sigma_{0} \in S(\Sigma):\left(\Omega, \Sigma_{0}, P\right)\right.$ has no atoms $\}$, the space of continuous sub- $\sigma$-algebras of $\Sigma$. Of course, if $\Sigma$ is not a continuous $\sigma$-algebra, then $S_{c}(\Sigma)=\emptyset$. On the other hand, if $\Sigma$ is continuous, then $S_{c}(\Sigma)$ is a very rich set to look at. For example, if $G$ is a group of $\Sigma$-measurable transformations of $\Omega$, let $\Sigma_{G}$ denote the $\sigma$-algebra of $G$-invariant sets in $\Sigma$. If $G$ is a finite group, then $\Sigma_{G} \in S_{c}(\Sigma)$. This provides a way in which to construct many interesting examples of elements of $S_{c}(\Sigma)$. In spite of this, $S_{c}(\Sigma)$ is a "small" subset of $S(\Sigma)$.

THEOREM 4. $S_{c}(\Sigma)$ is a closed nowhere dense subset of $S(\Sigma)$.

PROOF. We first observe that if $\Sigma_{0} \in S_{c}(\Sigma)$ and $A \in \Sigma$, then

$$
\left\{P(A \bigcap B): B \in \Sigma_{0}\right\}=[0, P(A)]
$$

Now fix $\Sigma_{1} \in S(\Sigma) \backslash S_{c}(\Sigma)$ and let $A$ be an atom of $\Sigma_{1}$. By the above observation, for any $\Sigma_{0} \in S_{c}(\Sigma)$, there exists $B \in \Sigma_{0}$ such that $P(A \cap B)=\frac{1}{2} P(A)$.

Let $f=\chi_{B}-\chi_{\Omega \backslash B} \in L_{1}^{\infty}\left(\Sigma_{0}\right)$. For any $g \in L_{1}^{\infty}\left(\Sigma_{1}\right), g$ is constant on $A$ and one easily checks that

$$
\|f-g\|_{2} \geq(P(A))^{1 / 2}
$$

Thus $d\left(\Sigma_{0}, \Sigma_{1}\right) \geq(P(A))^{1 / 2}$ for any $\Sigma_{0} \in S_{c}(\Sigma)$. Hence $S(\Sigma) \backslash S_{c}(\Sigma)$ is open and $S_{c}(\Sigma)$ is closed.

To see that $S_{c}(\Sigma)$ is nowhere dense, let $\Sigma_{0} \in S_{c}(\Sigma)$ and $\epsilon>0$. Choose $A \in \Sigma_{0}$ such that $0<P(A)<\epsilon^{2}$. Define $\Sigma_{1}$ to be the $\sigma$-algebra generated by $A$ and $\left\{B \in \Sigma_{0}: B \subset \Omega \backslash A\right\}$. Then $\Sigma_{1}$ has an atom $A$. For any $f \in L_{1}^{\infty}\left(\Sigma_{0}\right)$, let $g=\chi_{\Omega \backslash A} f \in L_{1}^{\infty}\left(\Sigma_{1}\right)$. Since $\|f-g\|_{2}^{2}=\int_{A}|f|^{2} \leq P(A)$ and $\Sigma_{1} \subset \Sigma_{0}$, we have that $d\left(\Sigma_{0}, \Sigma_{1}\right) \leq(P(A))^{1 / 2}<\epsilon$. Combined with the general estimate above, 
for this particular $\Sigma_{0}$ and $\Sigma_{1}, d\left(\Sigma_{0}, \Sigma_{1}\right)=(P(A))^{1 / 2}$. Thus the open ball of radius $\epsilon$ centered at $\Sigma_{0}$ is not contained in $S_{c}(\Sigma)$ for any $\epsilon>0$. Since $S_{c}(\Sigma)$ is closed, it is nowhere dense in $S(\Sigma)$.

Thus $\left(S_{c}(\mathbb{\Sigma}), d\right)$ is itself a complete metric space whose topological properties should be interesting to study. We do not consider such a study in this paper.

\section{CONNECTIONS WITH VON NEUMANN ALGEBRAS.}

There is a one-to-one correspondence between the sub- $\sigma$-algebras of $\Sigma$ and the von Neumann subalgebras of $L^{\infty}(\Omega, \Sigma, P)$. In this section, we assume the reader is familar with the basic theory of von Neumann algebras as found in a reference such as Sakai [7].

Let $\mathcal{M}$ be a fixed finite von Neumann algebra with a distinguished faithful, finite, normal trace $\tau$. For example, $\mathcal{M}$ could be $L^{\infty}(\Omega, \Sigma, P)$ or a Type $\mathrm{II}_{1}$-factor. Let $S(\mathcal{M})$ denote the set of all von Neumann subalgebras of $\mathcal{M}$ which have the same identity as $\mathcal{M}$.

In the case that $\mathcal{M}$ is a Type $\mathrm{II}_{1}$-factor, Christensen defined a metric on $S(\mathcal{M})$ in [2]. He showed that $S(\mathcal{M})$ then became a complete metric space. He used his metric to study perturbation properties of subfactors and his metric has also been used in the study of the index of a subfactor in a Type $\mathrm{II}_{1}$-factor (see [3] and [5]). Christensen's definition works well in the situation we are considering. For $x \in \mathcal{M}$, let $\|x\|_{2}=\sqrt{\tau\left(x x^{*}\right)}$. For $\mathcal{N}, \mathcal{L} \in \mathcal{S}(\mathcal{M})$, let $\mathcal{N}_{1}$ and $\mathcal{L}_{1}$ denote the unit balls in $\mathcal{N}$ and $\mathcal{L}$, respectively.

Then define

$$
d(\mathcal{N}, \mathcal{L})=\max \left\{\sup _{x \in \mathcal{N}_{1}} \inf _{y \in \mathcal{L}_{1}}\|x-y\|_{2}, \sup _{y \in \mathcal{L}_{1}} \inf _{x \in \mathcal{N}_{1}}\|x-y\|_{2}\right\}
$$

It is routine to check that $d$ is a metric on $S(\mathcal{M})$ and Christensen's proof that $S(\mathcal{M})$ is complete carries over to this more general situation.

Some of the proofs of section 3 can be adapted to the von Neumann algebra situation but not all. We list below what we can prove and leave the details of the adaption of the proofs to the interested reader.

THEOREM 5. Let $\mathcal{M}$ be a finite von Neumann algebra with a fixed faithful, finite trace $\tau$. With the Christensen's metric, $S(\mathcal{M})$ is compact if and only if $\mathcal{M}$ is generated by its minimal projections.

PROPOSITION 6. If $S(\mathcal{M})$ is connected, then $\mathcal{M}$ has at most one minimal projection.

OPEN PROBLEM Is the converse of proposition 6 true?

ACKNOWLEDGEMENT. This research was supported by an NSERC Canada operating grant.

\section{REFERENCES}

1. Boylan, E. S. Equiconvergence of Martingales, Ann. Math. Stat. 42 (1971), 552-559.

2. Christensen, E. Subalgebras of a Finite Algebra, Math. Ann. 243 (1979), 17-29.

3. Jones, V. F. R. Index for Subfactors, Invent. Math. 72 (1983), 1-25.

4. Kudo, H. A Note on the Strong Convergence of $\sigma$-algebras, Ann. Prob. 2 (1974), 76-83.

5. Mashhood, B. and Taylor, K. F. On Continuity of the Index of Subfactors of a Finite Factor, J. Func. Anal. 12 (1988), 56-66.

6. Rogge, L. Uniform Inequalities for Conditional Expectations, Ann. Prob. 2 (1974), 486-489.

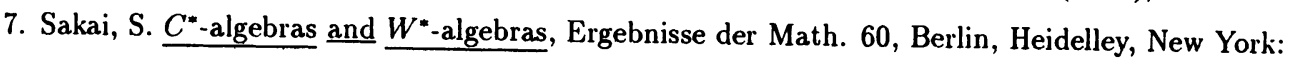




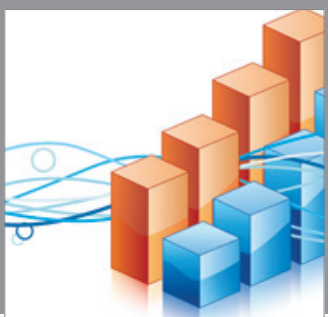

Advances in

Operations Research

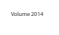

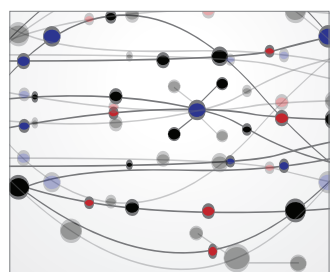

\section{The Scientific} World Journal
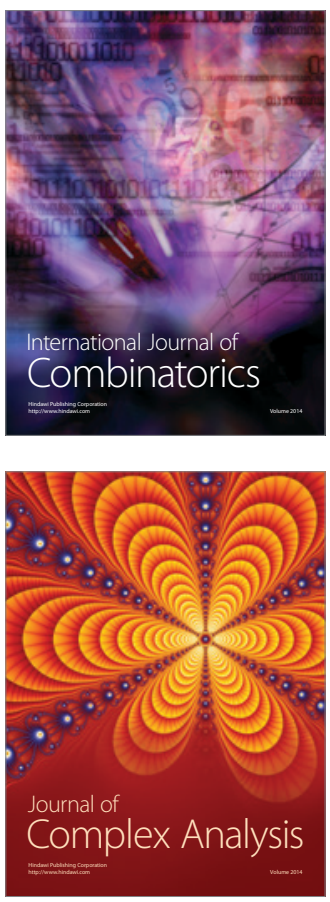

International Journal of

Mathematics and

Mathematical

Sciences
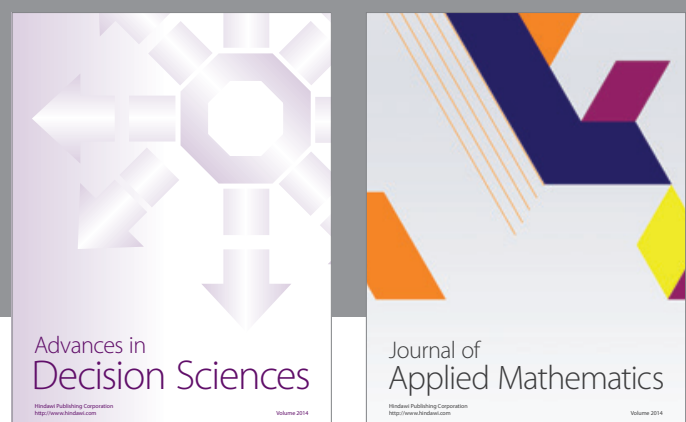

Journal of

Applied Mathematics
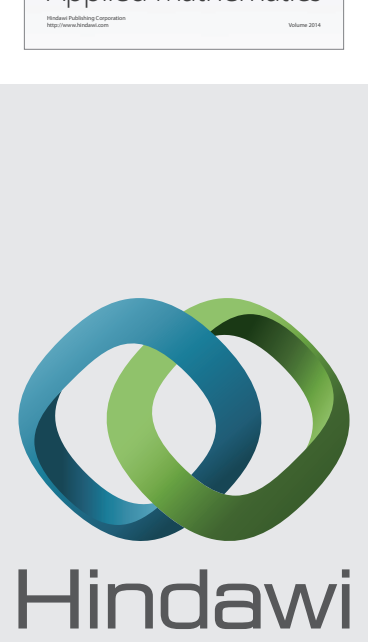

Submit your manuscripts at http://www.hindawi.com
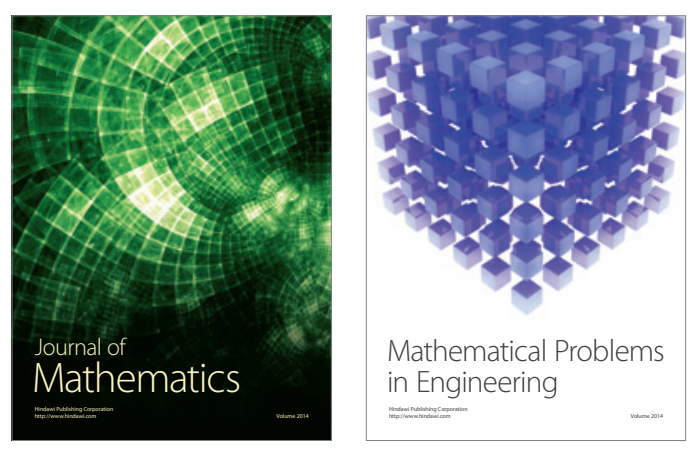

Mathematical Problems in Engineering
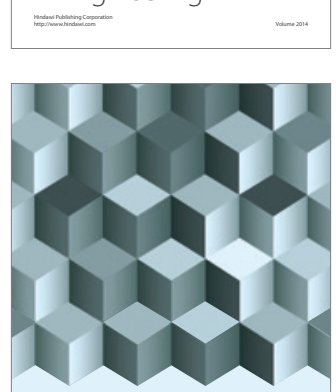

Journal of

Function Spaces
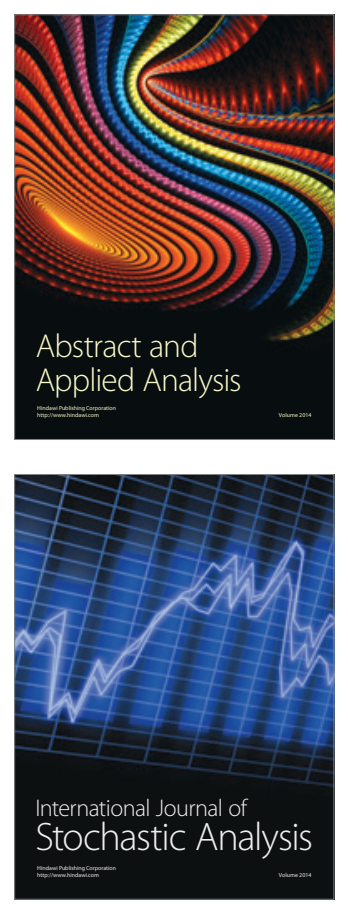

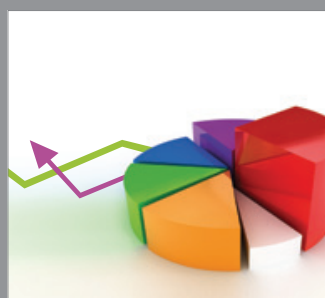

ournal of

Probability and Statistics

Promensencen
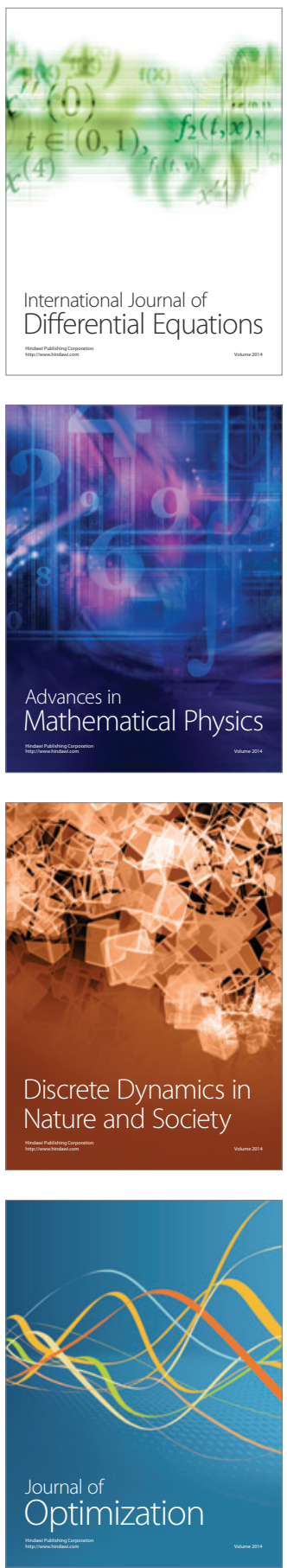